\title{
Responsabilidad Social Universitaria y ciudadanía democrática: la apuesta de formación para el compromiso público con las diversidades
}

\author{
University Social Responsibility and democratic citizenship: education's commitment \\ towards diversity public recognition
}

Lars Stojnic Chávez

Pontificia Universidad Católica del Perú

stojnic.lg@pucp.pe

Lima, Perú

\begin{abstract}
Resumen:
El artículo analiza si la experiencia universitaria incidiría en una mayor disposición de las y los estudiantes para comprometerse con la protección de las libertades y la convivencia pública entre subjetividades diversas, aspectos claves para la convivencia democrática. Recoge la apuesta del enfoque de Responsabilidad Social Universitaria por interpelar a la universidad como institución social que aporte a la formación de ciudadanos críticos, empáticos y comprometidos. Lo desarrollado es producto del recojo de información (tanto cuantitativo, como cualitativo) sobre la experiencia en un curso de una universidad peruana y del análisis de sus estrategias de aprendizaje, orientadas a fomentar que los y las estudiantes se involucren activamente en el diseño e implementación de propuestas de reflexión-acción sobre una problemática social particular. Los resultados evidencian que el curso tendría un efecto positivo y significativo en el aumento de la disposición de las y los estudiantes para reconocer la importancia de participar públicamente en favor de la protección de derechos de minorías sociales. En esa línea, se busca aportar en la discusión sobre el carácter distintivo de la responsabilidad social en los ámbitos universitarios, particularmente con respecto al fortalecimiento de una ética pública democrática.
\end{abstract}

Palabras clave: Educación universitaria; Responsabilidad Social; Educación para la Ciudadanía; Democracia; Compromiso Público.

\begin{abstract}
:
This article analyzes whether the university experience would influence a greater disposition of the students to commit to the protection of freedoms and public coexistence among diverse subjectivities, fundamental aspects of democratic coexistence. It connects the commitment of the University Social Responsibility approach to challenge the university as a social institution that contributes to the formation of critical, empathic and committed citizens. What is presented is the product of the collection of information (both quantitative and qualitative) about the experience of a subject in a Peruvian university and the analysis of its learning strategies aimed at encouraging students to be actively involved in the design and implementation of reflection-action proposals on a particular social problem. The results show that the experience in the subject would have a positive and significant effect on increasing the willingness of students to recognize the importance of participating publicly in favor of the protection of the rights of social minorities. Therefore we look to contribute in the discussion about the distinctive character of social responsibility in university settings, particularly with regard to strengthening a democratic public ethic.
\end{abstract}

Keywords: University education; Social Responsibility; Citizenship Education; Democracy; Public Engagement.

Pag. 32-47
Publicado:

05/01/2022 


\section{Responsabilidad Social Universitaria y ciudadanía democrática: la apuesta de formación para el compromiso público con las diversidades}

\section{| Introducción}

El artículo discute cómo la transversalización del enfoque de Responsabilidad Social Universitaria (RSU) en el quehacer de formación estaría fuertemente relacionado al desarrollo de aprendizajes significativos asociados al fortalecimiento de una ciudadanía democrática. De manera particular, se pone énfasis en el fortalecimiento de la disposición favorable de la ciudadanía hacia la protección pública de libertades y la promoción de una convivencia entre subjetividades diversas; aspectos fundamentales para ampliar las oportunidades de contestación de las relaciones de poder existentes, dentro de los marcos de una convivencia democrática (Mouffe, 1999).

En un contexto como el latinoamericano, marcado por amplias brechas de desigualdad e injusticia social, la RSU tiene el potencial de promover una nueva forma de aproximarse a la dirección y gestión de las universidades, aportando particularmente en el fortalecimiento de su rol para promover modelos de desarrollo sostenible y justo. Deben, así, reconocerse como organizaciones con poder, "por su capacidad de interactuar en la sociedad de la que forma parte [y por su] profunda orientación transformadora" (De La Cruz \& Sasia, 2008, p.24) y, por tanto, el enfoque tendría el potencial de interpelar la identidad y legitimidad de las universidades con respecto al cumplimiento efectivo de sus quehaceres fundamentales (Vallaeys, 2014).
En tal sentido, es importante ahondar en la discusión sobre cómo se concreta la conexión entre el quehacer de formación de la Universidad y el enfoque de RSU, debido a los desafíos que la enfrenta como institución social. En las últimas décadas la educación superior ha adoptado una orientación fuertemente marcada por las demandas del mercado y de los sistemas productivos por una fuerza laboral de mayor calidad (Cuenca, 2018; Chiroleu, 2011; Gasca-Pliego \& Olvera-García, 2011; White, 2013) y dicho énfasis profesionalizante, se aleja o anula el potencial de la educación superior para la formación de ciudadanos y profesionales que logren reconocer la realidad que les rodea y poder comprometerse con la transformación de situaciones y problemas. Por eso es indispensable que las universidades asuman la encrucijada de sus responsabilidades, que las obliga -entre otros aspectos"a reflexionar sobre qué ciudadanía forman y para qué tipo de sociedad" (Evia, et.al., 2017, p.81).

Esta discusión cobra relevancia en el caso peruano, por un lado, debido a los planteamientos de la nueva Ley Universitaria $\mathrm{N}^{\circ} 30220$ (2014), la cual destacó por primera vez la relevancia de la RSU como fundamento de la vida universitaria, asociándola con el compromiso con una formación con "pleno sentido de responsabilidad social con las necesidades del país...[y con] la afirmación de la democracia, el estado de derecho y la inclusión social" (Ley N³0220, 2014) -en sus art.6 y art. 124-. En tal sentido, se plantea un marco de referencia normativo que realza la importancia de fortalecer el compromiso de las universidades -como destaca Vallaeys (2008, p. 209) 
- con una “...formación de ciudadanos responsables y solidarios".

Por otro lado, en las evidencias sobre como la promoción del enfoque de RSU en las universidades peruanas no se estaría dando principalmente en relación al quehacer de formación, se encuentra el estudio denominado Investigación Continental: Estado del arte de la RSU en América Latina 2019 (Unión de Responsabilidad Social Universitaria Latinoamericana [URSULA], 2019), se muestra que el promedio de las universidades peruanas está por debajo del promedio general en metas como la promoción de iniciativas de aprendizaje en servicio y de inclusión curricular de los Objetivos de Desarrollo Sostenible (ODS). Mientras que el balance en La Responsabilidad Social en el Perú: aproximación inicial 2016-2017 (Stojnic \& Jungbluth, 2018) muestra que sólo el $10 \%$ de las 50 universidades que fueron parte del estudio reconocían como una función formal la incorporación del enfoque en las tareas formativas y que solo el $26 \%$ asoció la promoción del enfoque con la posibilidad de apostar por una formación ciudadana para el desarrollo social.

Así pues, es importante seguir fortaleciendo la discusión en torno a lo que significa que una universidad se conciba como socialmente responsable, poniendo énfasis en la interpelación de su función social de formación. Particularmente, ahondando la discusión sobre el aporte de las universidades para la expansión y el fomento de prácticas y comportamientos democráticos, así como de una cultura pública de ejercicio y convivencia ciudadana (Jelin, 1996 como se cita en Gasca-Pliego \& Olvera-García, 2011), en tanto, la adhesión pública de la ciudadanía a las instituciones, formas de convivencia y principios fundamentales de la democracia sería un componente fundamental para fortalecer la legitimidad del sistema.

En esa línea, diversos estudios destacan la importancia de la universidad para promover el desarrollo de actitudes consideradas sustantivas en el sostenimiento del sistema y convivencia democrática (Booth \& Seligson, 2009; Golebiowska, 1995; Juliá, 2015; Martínez, 2006); como el reconocimiento de los derechos de las minorías a participar de la vida política (Carrión, et.al., 2020; Stojnic \& Román, 2016) y de la propia competencia para participar activamente en favor de un bienestar asociado a la inclusión pública y política (Carrión, et.al., 2012; Morrell, 2005; Pérez, 2009; Stojnic 2016). Al respecto, es posible identificar dos aproximaciones sobre cómo desde la RSU se conectaría la formación ciudadana como aspecto fundamental del quehacer de formación universitaria.

La primera, destaca el ejercicio de la ciudadanía como un aspecto de la formación para el ejercicio profesional. Se trataría así, de "formar ciudadanos capaces de ejercer su profesión con sentido de responsabilidad" (Salazar, 2003, p. 32) y de que "durante la trayectoria académica el estudiantado sea consciente de su...papel como profesional en la sociedad" (Evia, et.al., 2017, p.101). En tal sentido, la responsabilidad pública de la universidad radicaría en aportar a que sus estudiantes "ejerzan sus futuras profesiones con la voluntad de contribuir a la formación de una sociedad inclusiva, digna y democrática" (Martínez, 2006, p.87).

La segunda reconoce a la ciudadanía como una identidad que -más que subsumirse a- interactúa con la identidad profesional y, por tanto, la principal preocupación estaría en interpelar los procesos de formación de la universidad para que "influyan e incluso formen el juicio éticociudadano de sus estudiantes...sobre todo en el papel que ejerzan en la esfera de lo público y en la construcción de un sistema democrático" (Gasca-Pliego \& Olvera-García, 2011, p.51).

Ahora bien, desde ambas perspectivas se identifican coincidencias con respecto a conectar la formación con la apuesta en favor de la ciudadanía y cómo esta debería ser 
incorporada curricularmente (Evia, et.al, 2017; Martínez, 2006; Salazar, 2003). Se destaca, en dicho sentido, el valor de que se promuevan propuestas pedagógicas que permitan conectar los ámbitos disciplinares con la realidad social $\mathrm{y}$, por tanto, fomentar aprendizajes significativos $\mathrm{y}$ situados en el contexto del estudiantado (Evia, et.al, 2017). Así mismo, que se fomenten experiencias prácticas en el currículo formal, así como en programas y acciones pedagógicas, orientados explícitamente al “desarrollo de estructuras cognitivas y afectivas...[que ofrezcan] a los estudiantes todas las oportunidades posibles de desarrollar los atributos necesarios para ser ciudadanos competentes" (Salazar, 2003, p. 41). Como las capacidades y disposiciones para comprometerse e implicarse, de escuchar y ponerse en lugar del otro, de pensar en forma crítica y empática y de preocuparse no solo por su bienestar sino por el bienestar de todos los que lo rodean (Arango et.al. 2014; Martí, et.al. 2014).

Partiendo de lo desarrollado, el presente trabajo tiene como propósito analizar si es que la experiencia de las y los estudiantes en un curso universitario diseñado en concordancia con las apuestas antes señaladas, sería un factor de incidencia en el desarrollo de una disposición favorable para involucrarse en la defensa de derechos y libertades de subjetividades no hegemónicas. Se propone, en tal sentido, un estudio de caso en dos etapas, una de corte cuantitativa y otra cualitativa.

El objetivo de la primera, fue cuestionar si el simple hecho de avanzar en la educación superior incidiría en el desarrollo de disposiciones favorables hacia la democracia, o si más bien, sería importante analizar los procesos y la experiencia educativa como dimensiones fundamentales. En tal sentido, las hipótesis puestas a prueba fueron que: "La experiencia en un curso universitario orientado explícitamente a que las y los estudiantes interpelen el ejercicio de su ciudadanía -a diferencia del número de semestres cursados:
H1: ...tendría un efecto positivo en su disposición a reconocer la participación activa como un componente importante para el ejercicio de la ciudadanía en democracia".

H2: ...tendría un efecto positivo en su disposición a reconocer la importancia de involucrarse en protestas pacíficas en favor de proteger las libertades de minorías sociales".

El objetivo de la segunda etapa (de corte cualitativo), fue indagar, partiendo de los hallazgos de la primera, por un lado, en qué medida las y los estudiantes consideraban que su experiencia en el curso había incidido positivamente en el desarrollo de las disposiciones analizadas; y por otro lado, identificar aquellos aspectos de su experiencia que pudiesen aportar elementos para entender los cambios identificados.

\section{| Método}

La información aprovechada en el presente trabajo surgió de un estudio de caso, particularmente de la experiencia de un curso de una universidad peruana, ubicada en la ciudad de Lima y de gestión privada. La selección de la universidad radicó, por un lado, a su apuesta institucional en favor de la RSU como dimensión importante en la gestión y gobierno universitario, incluso varios años antes de la promulgación de la Ley Universitaria en el Perú en que se planteó como obligatoria; lo cual derivó en que sea una de las primeras universidades peruanas que ha optado por incorporar el enfoque de RSU en el nivel curricular y, por tanto, reflejado en sus planes de estudio. Por otro lado, debido a la orientación histórica de dicha universidad por fomentar una formación básica 
integral, interdisciplinaria y humanista; debido a lo cual, en sus primeros dos años de estudios las y los estudiantes toman cursos de diversa índole -no necesariamente relacionados a los estudios de pre-grado especializados que seguirán posteriormente- y que buscan incidir en dicho sentido.

El curso analizado -que denominaremos 'Ciudadanía'fue uno de los cursos precursores de dicha apuesta institucional, y al tener carácter obligatorio-optativo las y los estudiantes cursando esta etapa universitaria lo podían elegir dentro de una de las áreas del plan de estudios. El curso estaba orientado a promover la competencia genérica 'ética y ciudadanía' y en esa línea, tenía como propósito motivar a los estudiantes a reflexionar sobre la expansión de la ciudadanía en el Perú, y el ejercicio de su propia ciudadanía, desde una perspectiva teórica y práctica. En tal sentido, promovía que las y los estudiantes se aproximasen a diversos problemas sociales (como la discriminación y la desigualdad social) desde una perspectiva histórica y crítica, y en diálogo permanente con tres enfoques base del curso: ciudadanía democrática, responsabilidad social y desarrollo humano.

Para abordar los objetivos trazados se optó por el recojo y análisis de información combinando una aproximación cuantitativa y cualitativa. La primera, con el propósito de analizar posibles cambios en las disposiciones ciudadanas examinadas de las y los estudiantes al inicio y final de su experiencia en el curso, contrastándolo con el efecto de los semestres acumulados y, por tanto, analizar las hipótesis trazadas. La segunda, y en caso de que se corroborasen las hipótesis, tenía el propósito de indagar sobre la experiencia de las y los estudiantes en el curso, buscando identificar aquellos aspectos del mismo que pudiesen relacionarse con los cambios identificados.

Así, y como primera etapa del proceso de recojo de información, se aplicó una encuesta al inicio y final de los dos semestres del año académico 2017. Se administró en todos los horarios del curso, cada uno de los cuáles contaba con 60 estudiantes matriculados. Para evaluar la validez y fiabilidad del instrumento se realizó el análisis del coeficiente Alfa de Cronbach en todos los ítems del instrumento, dando como resultado 0.849 , lo que indica un buen nivel de confiabilidad.

La encuesta empleada tenía un total de 47 preguntas cerradas con opciones a elegir y estaba estructurada en tres partes, con el propósito de recoger información sobre: 1) disposiciones asociadas a la institucionalidad y convivencia democrática (se emplearon escalas Likert); 2) experiencia educativa; y 3) aspectos sociodemográficos (sexo y tipo de escuela secundaria a la que asistieron) y aspectos familiares (nivel educativo de padres, su involucramiento en asociaciones y experiencia familiar discutiendo sobre temas políticos) .

En el primer semestre del 2017 se completó la encuesta con100 estudiantes al inicio del curso y 104 al cierre (correspondiente a los dos horarios que se dictaron); y durante el segundo, 158 lo hicieron al inicio y 128 al final (correspondiente a los tres horarios dictados). En ambos semestres, la encuesta se aplicó durante la primera y última semana de clases, a todas y todos aquellos estudiantes que asistieron a las sesiones; y debido a que para el análisis no se haría distinción entre ambos semestres, el armado de la base de datos se hizo agrupando las respuestas de los dos grupos, por lo que se considera un total de 268 encuestas completadas al inicio del curso y 232 al cierre. En la siguiente tabla se presenta información sobre la muestra con respecto a variables socio demográficas de las y los encuestados, así como del semestre en el que se encontraban cuando llevaron el curso (Tabla 1). 
Tabla 1

Caracterización de la muestra

\begin{tabular}{|c|c|c|c|c|}
\hline & & & $\begin{array}{c}\text { Inicio del Semestre } \\
(2017-1 \text { y 2017-2) }\end{array}$ & $\begin{array}{c}\text { Final del Semestre } \\
(2017-1 \text { y 2017-2) }\end{array}$ \\
\hline \multirow{4}{*}{ 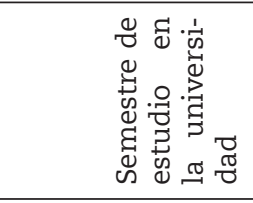 } & Primero & & $1(0.40 \%)$ & $0(0.0 \%)$ \\
\hline & Segundo & & $72(28.80 \%)$ & $55(24.23 \%)$ \\
\hline & Tercero & & $80(32 \%)$ & 86 (37.89\%) \\
\hline & Cuarto & & 97 (38.80\%) & $86(37.89 \%)$ \\
\hline Total & & & 250 & 27 \\
\hline \multirow{2}{*}{ 䒽 } & Mujer & & $161(62.89 \%)$ & $150(65.79 \%)$ \\
\hline & Hombre & & $94(36.72 \%)$ & 78 (34.21\%) \\
\hline Total & & & 255 & 228 \\
\hline \multirow{3}{*}{ 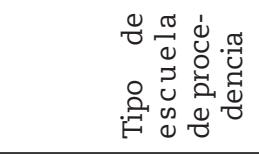 } & Pública & & 35 (13. 38\%) & $30(13.22 \%)$ \\
\hline & Privada Religiosa & & $108(42.19 \%)$ & $92(40.53 \%)$ \\
\hline & Privada Laica & & $114(44.53 \%)$ & $105(46.26 \%)$ \\
\hline \multirow{5}{*}{ Total } & & & 256 & 227 \\
\hline & \multicolumn{4}{|c|}{ Padre } \\
\hline & Primaria & Incompleta & $2(0.83 \%)$ & $2(0.92 \%)$ \\
\hline & & Completa & $4(1.65 \%)$ & $1(0.46 \%)$ \\
\hline & Secundaria & Incompleta & $5(2.07 \%)$ & $1(0.46 \%)$ \\
\hline \multirow{14}{*}{ 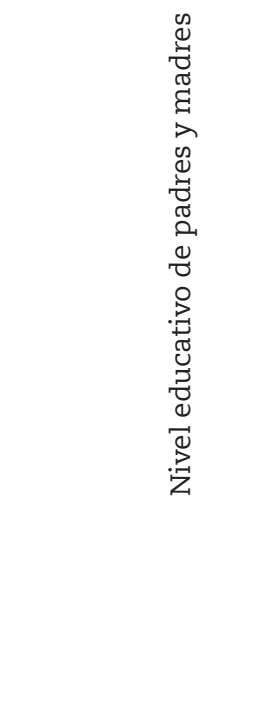 } & & Completa & $30(12.4 \%)$ & 35 (16.13\%) \\
\hline & Superior & Incompleta & $42(17.36 \%)$ & 35 (16.13\%) \\
\hline & & Completa & $151(62.4 \%)$ & $138(63.59 \%$ \\
\hline & No sabe & & $8(3.31 \%)$ & $5(2.3 \%)$ \\
\hline & Total & & 242 & 220 \\
\hline & \multicolumn{4}{|c|}{ Madre } \\
\hline & Primaria & Incompleta & $1(0.41 \%)$ & $1(0.46 \%)$ \\
\hline & & Completa & $1(0.41 \%)$ & $2(0.91 \%)$ \\
\hline & Secundaria & Incompleta & $2(0.82 \%)$ & $5(2.28 \%)$ \\
\hline & & Completa & $28(11.52 \%)$ & $32(14.61 \%)$ \\
\hline & Superior & Incompleta & $53(21.81 \%)$ & $46(21 \%)$ \\
\hline & & Completa & $153(62.96 \%)$ & 131 (59.82\%) \\
\hline & No sabe & & $5(2.06 \%)$ & $2(0.91 \%)$ \\
\hline & Total & & 243 & 219 \\
\hline
\end{tabular}

Nota: Elaboración propia.

Para analizar las hipótesis señaladas sobre la relación entre la experiencia universitaria y las disposiciones antes descritas, se aprovecharon:

- Tres variables dependientes: i) valoración de la participación ciudadana activa, en contraposición a una meramente electoral, como característica fundamental de democracia; ii) reconocimiento de las protestas pacíficas en caso de que los derechos de minorías sociales se vieran afectadas; $\mathrm{y}$, iii) disposición a involucrarse en movilizaciones pacíficas para garantizar derechos de una minoría social.

- Dos variables independientes: i) 'curso ciudadanía', que da cuenta del posible efecto de la experiencia de las y los estudiantes en el mismo; y ii) 'avanzar en la universidad', que analiza el posible efecto del tiempo (número de semestres) en la universidad. 
- Nueve variables de control: i) dos disposiciones consideradas claves en los estudios sobre actitudes democráticas (valoración de la democracia e interés en la política); ii) tres variables socio-demográficas (sexo, nivel económico y colegio secundario de procedencia); y, iii) cuatro variables asociadas a la experiencia familiar de las y los estudiantes (nivel educativo de padres, su involucramiento en asociaciones civiles y políticas y experiencia familiar discutiendo sobre temas políticos).

Los resultados cuantitativos que se presentan en la siguiente sección dan cuenta, por un lado, del análisis bivariable usado para identificar los cambios entre las disposiciones de las y los estudiantes al inicio y cierre de cada semestre. Por otro lado, se presentan modelos de regresión -bajo el método de Mínimos Cuadrados Ordinarios (MCO)- con el propósito de identificar la dirección e intensidad de las relaciones entre variables dependientes e independientes. Es importante destacar que todas las variables incluidas en el análisis para este artículo fueron recodificadas de 0 a $1^{\text {iii }}$-ya sea como escalas continuas o como variables dicotómicas-, con el propósito de simplificar el análisis estadístico y facilitar la lectura comparada de los coeficientes de los modelos de regresión múltiple.

Como segunda etapa del recojo de información, y desde una aproximación metodológica cualitativa, se aplicó una entrevista estructurada a un total de diez estudiantes hacia el final de su experiencia en el curso. Las y los estudiantes fueron seleccionados -al final de ambos semestres- por recomendación de los docentes, tomando en cuenta, criterios de asistencia y participación; y se optó por estudiantes que cursaban entre su segundo y cuarto semestre universitario, con el propósito de que pudiesen responder las preguntas teniendo al menos dos semestres de experiencia universitaria.

La entrevista estuvo dirigida a conocer, por un lado, la consideración de las y los estudiantes con respecto a los principales aprendizajes desarrollados en el curso a lo largo del semestre y, por otro lado, indagar sobre su valoración con respecto a una de las estrategias del curso, denominada 'Perfil de Intervención'. Dicha estrategia, fundamental en el desarrollo del curso, se orientaba, por un lado, a que las y los estudiantes indaguen sobre una problemática y desarrollen su vínculo con los tres enfoques transversales del curso. Por otro lado, a que en grupos profundicen un caso específico de dicha problemática y trabajen en el diseño de actividades para compartir las reflexiones, interpelaciones y sentires desarrollados a lo largo del semestre -como hito de cierre de la estrategia-, en un espacio denominado 'Feria'. En dicho espacio, y a modo de intervención en el campus de la Universidad, las y los estudiantes presentaban los recursos y estrategias diseñadas para la sensibilización y el debate sobre los temas abordados a la diversidad del público participante.

Así, el propósito del análisis cualitativo fue complementar los hallazgos del análisis cuantitativo, sobre la posible relación entre la experiencia del curso y las disposiciones abordadas, así como plantear una primera aproximación para comprender qué elementos de las estrategias desplegadas en el propio curso podrían permitir comprender las relaciones identificadas.

\section{| Resultados}

El análisis desarrollado apunta a brindar evidencia que permita, desde la perspectiva del enfoque de RSU, ampliar el debate sobre la apuesta por una formación de calidad en las instituciones universitarias. Particularmente discutiendo la noción de que avanzar en el sistema educativo -entendido como el acceso y acumulación de semestres/años de educación superior"automáticamente" incidiría en una educación de calidad y asociada al desarrollo de actitudes que favorezcan el 
fortalecimiento de una vivencia social de la democracia.

En primer lugar, y partiendo del análisis cuantitativo realizado, se presentan los resultados asociados a la relación entre la experiencia en el curso y las disposiciones analizadas. Así mismo, se complementan los hallazgos con testimonios recogidos de las y los estudiantes; para mantener su anonimato, luego de cada testimonio se indica un código formado por la palabra 'Entrevistada' y seguido por un número.

Con respecto al reconocimiento de la participación ciudadana como un componente fundamental de la democracia por parte de los y las estudiantes, los resultados presentados en la Tabla 2 evidencian un incremento positivo y significativo (al 99\%) de la media entre el inicio y el final del curso.

Tabla 2

Prueba t-student entre valoración de la participación ciudadana activa y curso 'Ciudadanía'

\begin{tabular}{ccccc}
\hline Media antes del curso & Media después del curso & T & DF & P \\
\hline 0.519 & 0.636 & -4.0839 & 442 & 0.0001 \\
\hline
\end{tabular}

Nota: Elaboración propia.

Esta relación se ve reforzada por el modelo de regresión (Tabla 3), en el que -controlando por las demás variablesse evidencia que la experiencia en el curso incidiría positivamente a un $99 \%$ de confianza, mientras que la

Tabla 3

Modelo de regresión valoración de la participación ciudadana activa

\begin{tabular}{lc}
\hline \multicolumn{1}{c}{ Variables } & $0,06(, 06)$ \\
\hline Apoyo a la idea general de la democracia & $0,05(, 04)$ \\
\hline Tipo de colegio & $0,06(, 04)$ \\
\hline Sexo & $-0,08(, 06)$ \\
\hline Nivel económico & $0.17(, 09)+$ \\
\hline Interés en política & $0,01(, 09)$ \\
\hline Avanzar en la universidad & $0,14(, 03)^{* * *}$ \\
\hline Curso 'Ciudadanía' & $0.03(, 12)$ \\
\hline Nivel educativo de padre y madre & $-0,09(, 08)$ \\
\hline Discusiones sobre temas sociales y políticos en familia & $-0.12(, 05)^{* * *}$ \\
\hline Participación de padre y/o madre en asociaciones políticas & $0.06(, 04)$ \\
\hline Participación de padre y/o madre en asociaciones civiles & $0,37(, 15)^{*}$ \\
\hline Constante & $0,11^{* * *}$ \\
\hline R2 & 322 \\
\hline N & \\
\hline
\end{tabular}

Nota: Leyenda de significancia: $+\mathrm{p}<0.1 ;{ }^{*} \mathrm{p}<.05 ;{ }^{* *} \mathrm{p}<.01 ;{ }^{* * *} \mathrm{p}<.001$

Esta relación se puede comprender mejor, en las propias voces de las y los estudiantes, quienes al ser consultados sobre los aprendizajes desarrollados durante el curso variable 'avanzar en la vida universitaria' no presenta ninguna influencia con respecto a la disposición analizada. destacaron su influencia para reconocer la importancia del involucramiento activo de la ciudadanía en favor del desarrollo y bienestar colectivo. 
“...nunca había hecho caso al ámbito público, y para mí era algo sabido que existe corrupción...entonces, [el curso] ha logrado que concientice que también soy parte de esa elección, ya que [incluso] sin hacer nada también tengo responsabilidad social acerca de eso...también tengo poder" (Entrevistada 5).

“...personalmente el curso me ha sido de gran utilidad para reformularme varias ideas...por ejemplo, que se entiende por ciudadanía, como debería ser el ideal del comportamiento de un ciudadano en el grupo; que tiene que ser más activo y no sólo...actuar de manera pasiva, sólo ir a votar y de ahí acaba todo, sino demandar al Estado cosas, relacionarse con los otros (Entrevistada 4).

Como se evidencia en ambos testimonios, los estudiantes reconocen las posibilidades que pudieron experimentar para, por un lado, cuestionarse sobre el significado de ejercer la propia ciudadanía y por otro lado, para reforzar su auto reconocimiento como sujetos con capacidad de incidencia pública para reproducir estructuras sociales 0 transformarlas.

Con respecto a las disposiciones de las y los estudiantes para reconocer el valor de las protestas como medidas legítimas ante posibles restricciones de las libertades de grupos socialmente minoritarios, así como para participar en manifestaciones públicas en favor de los derechos de dichos grupos, los resultados de la Tabla 4 también evidencian un aumento entre la media al inicio y el final del curso. El análisis da cuenta de una relación positiva y significativa (al 95\% de confianza) entre la experiencia en el curso y el incremento de ambas disposiciones.

Tabla 4

Pruebas T-student sobre disposiciones de estudiantes al inicio y cierre del curso 'Ciudadanía'

Antes del curso Después del curso

Para reconocer protestas pacíficas como legítimas cuando libertades de comunidades indígenas y LGTBIQ son restringidas
0.685

0.761

487

0.0022
Para participar de protestas pacíficas en favor de derechos de grupos minoritarios
0.635

0.730

$-3.2666$

478

0.0012

Nota: Elaboración propia

Así mismo, y de manera complementaria, los modelos de regresión que se presentan en la Tabla 5 refuerzan (a un 99\% de confianza) la relación de influencia del curso con respecto a una mayor disposición para reconocer la importancia de la participación pública para limitar posibles manifestaciones de opresión ante diversas minorías. 
Tabla 5

Modelos de regresión sobre disposición de estudiantes

\begin{tabular}{|c|c|c|}
\hline Variables & $\begin{array}{l}\text { Para reconocer protestas pacíficas como le- } \\
\text { gítimas cuando restringen libertades }\end{array}$ & Para participar de protestas pacíficas \\
\hline Apoyo a idea general de democracia & $0,11(, 05)^{*}$ & $0,07(, 06)$ \\
\hline Tipo de colegio & $0,10(, 03)^{* * *}$ & $0,01(, 03)$ \\
\hline Sexo & $0,06(, 03)^{*}$ & $0,24(, 03)^{* * *}$ \\
\hline Nivel económico & $-0,09(, 05)^{*}$ & $-0,04(, 05)$ \\
\hline Interés en política & $0.22(, 07)^{* *}$ & $0.18(, 08)^{*}$ \\
\hline Avanzar en la universidad & $0,001(, 07)$ & $-0,17(, 07)^{*}$ \\
\hline Curso ciudadanía & $0,08(, 03)^{* *}$ & $0,11(, 03)^{* * *}$ \\
\hline Nivel educativo de padre y madre & $0.02(, 09)$ & $0.13(, 11)$ \\
\hline $\begin{array}{l}\text { Discusiones sobre temas sociales y políti- } \\
\text { cos en familia }\end{array}$ & $-0,05(, 06)$ & $-0,07(, 07)$ \\
\hline $\begin{array}{l}\text { Participación de padre y/o madre en aso- } \\
\text { ciaciones políticas }\end{array}$ & $0.01(, 04)$ & $0.04(, 04)$ \\
\hline $\begin{array}{l}\text { Participación de padre y/o madre en aso- } \\
\text { ciaciones civiles }\end{array}$ & $0.03(, 04)$ & $0.08(, 04)^{*}$ \\
\hline Constante & $0,44(, 12)^{* * *}$ & $0,34(, 14)^{*}$ \\
\hline R2 & $0,12^{* * *}$ & $0,23^{* * *}$ \\
\hline $\mathrm{N}$ & 352 & 352 \\
\hline
\end{tabular}

Nota: Leyenda de significancia: $+\mathrm{p}<0.1 ;{ }^{*} \mathrm{p}<.05 ;{ }^{* *} \mathrm{p}<.01 ;{ }^{* * *} \mathrm{p}<.001$

Así también, y de igual forma que en la tabla 3, los modelos evidencian que el aumento de dichas disposiciones no tendría relación con la acumulación de semestres universitarios; llamando particularmente la atención que en el segundo modelo la variable 'avanzar en la universidad' incluso tendría una relación negativa .

Ahora bien, a partir de lo recogido en los testimonios de las y los estudiantes, es posible complementar el hallazgo destacando el valor que le otorgan a como la experiencia en el curso habría incidido en una aproximación a las problemáticas abordadas desde una perspectiva más humana y con mayor reconocimiento de la interconexión entre la realidad, su vivencia propia y la de diversos grupos humanos. “...yo venía de una familia que se podía considerar un poco conservadora y rígida, que ven el desarrollo como algo más económico y este curso como que ha podido posibilitar ampliar mi panorama sobre lo que es desarrollo, incentivando más, haciendo hincapié más en el ser humano, más que en un desarrollo económico" (Entrevistada 4).

“...como si a mí me movieran de mi casa porque hay algo más importante que yo supuestamente; es como que destruyen toda mi vida solo [por] un apoyo económico mayor...me di cuenta que mucha gente estaba siendo vulnerada debido a esto, y que hay gente como nosotros que no nos damos cuenta, o que incluso apoyamos la idea. Y creo que gracias 
[al curso] me di cuenta que era yo la que tenía que cambiar" (Entrevistada 3).

Incluso, en el segundo testimonio -en que se refiere al efecto de la minería en el desplazamiento de diversas comunidades- se evidencia el cuestionamiento de la estudiante con respecto a su manera de aproximarse a nociones como riqueza y desarrollo, y el cambio hacia una perspectiva en que el bienestar de las personas se coloque por delante.

En segundo lugar, y poniendo el énfasis en el análisis cualitativo realizado, se presentan los resultados que permiten ahondar en cómo la experiencia en el curso -a partir de la interacción de las y los estudiantes con su organización y estrategias- incidiría en el desarrollo y fortalecimiento de las disposiciones analizadas. Los hallazgos, organizados en tres aspectos, se ilustran a partir de fragmentos de los testimonios surgidos del análisis de las entrevistas.

Por un lado, es posible realzar la valoración de las y los estudiantes sobre la oportunidad que la organización del curso les brindó, a partir de la combinación de diversas estrategias para aproximarse de una manera más sentida a los temas y problemáticas abordados por el curso. Destaca el reconocimiento de las y los estudiantes sobre su experiencia involucrándose de manera práctica en el análisis y reflexión sobre diversas problemáticas; y cómo, al no haber sido una aproximación sólo teórica, sino crítica y con un sentido afectivo -en tanto, pudiendo aproximarse a la vivencia humana-, les permitió el desarrollo de aprendizajes significativos.

“...creo que lo que te deja más es lo que tú cambias, lo que tú sientes al trabajar estos temas...cualquier curso te puede dar información, pero creo que es muy diferente...[desarrollar] tu punto de vista, qué es esto, por qué es esto... sientes que estás en contacto más con los temas..." (Entrevistada 3). “...porque uno puede ampliar su panorama sobre lo que es desarrollo, que es libertad...y no solamente es teórico, sino que de manera práctica y participativa uno logra que los conceptos se queden...en uno (Entrevistada 4).

Así también, destaca la asociación que hacen entre su experiencia en el curso y su posibilidad de reconocer el cuidado de la diversidad subjetiva como un aspecto fundamental para el bienestar ciudadano. Más aún, su reconocimiento sobre la importancia de interpelarse como agentes, con el potencial de involucrarse -a partir de reconocerse interconectados con su entorno- en procesos de transformación para revertir problemáticas asociadas con el rechazo a las diferencias.

“...el curso me ha [ayudado a]...reformularme [sobre] el comportamiento de un ciudadano en el grupo...[al] relacionarse con los otros tratar de evitar, principalmente, la violencia hacia el otro, hacia lo distinto." (Entrevistada 4).

“...porque he podido rescatarme como persona a partir de los temas por ejemplo de discriminación... [reconocer que] soy también un agente social y soy parte de esta sociedad y que no estoy excluida, o no solamente soy yo, y también influyo, también tengo poder y también tengo acción social" Entrevistada 5.

Por otro lado, las y los estudiantes destacaron la orientación del curso hacia una acción reflexiva y sentida, en tanto, les habilitase la oportunidad de desarrollar elaboraciones (sobre problemáticas particulares) que las y los interpelasen y que les permitiese actuar sobre su entorno cercano, a partir de las mismas.

Los testimonios evidencian su valoración sobre cómo las estrategias del curso les permitieron visibilizar la importancia de dichas problemáticas en la cotidianeidad social, pero poniendo énfasis en su propio rol como 
ciudadanas y ciudadanos. Incluso con respecto a temas que previamente les podían haber sido ajenos, los testimonios muestran cómo la orientación del curso para la indagación de los mismos habría incidido en que se cuestionen e interpelen sobre su responsabilidad cotidiana en la reproducción de dichas problemáticas y de sus consecuencias.

"Muchos de los temas que hemos tratado han sido controversiales y la forma en la que hemos hablado de éstos...además, tenemos una feria de intervenciones, que gracias a toda la información que hemos logrado recabar ha hecho que en este perfil -yo que traté el tema de discriminación- me di cuenta de que es más difícil que las personas que no sabemos de esto sepan reconocer que en realidad son agentes de cambio...[de que] somos capaces de tratarlos" (Entrevistada 3).

“...he podido...concientizar que existen los problemas [como la discriminación], los sabemos, pero no logramos...o no queremos analizarlos, o sentimos que somos externos...cuando no es cierto, [cuando] es posible que nosotros también al decidir no involucrarnos también estemos optando porque...se sigan reproduciendo" (Entrevistada 5).

En la misma línea, se identifica la valoración de las y los estudiantes sobre cómo -particularmente con respecto a las estrategias del Perfil de Intervención y de la Feria-habrían tenido la oportunidad de orientar dichas interpelaciones hacia la formulación de propuestas que, al ser llevadas a la práctica, les permitió compartir en el contexto de su comunidad universitaria sus ideas, reflexiones, cuestionamientos y sentires.

"Es un curso innovador...te traen a una Feria para que presentes lo que has ido desarrollando...[y] comienzas a notar que tú tienes poder para poder realizar acciones y poder realizar cambios en nuestra sociedad...y dentro de la Universidad" (Entrevistada 2).

“...uno de los aspectos que más me gustó fue llegar a la gente, visibilizar una problemática que muchas veces es ignorada, y causar un impacto, una reflexión...la gente puede decir que es mínimo, y puede ser, pero por algo se empieza" (Entrevistada $6)$.

Así, en los testimonios se evidencia el reconocimiento de las y los estudiantes sobre las posibilidades que el curso les brindó para fortalecer su auto reconocimiento como sujetos de poder público. Concretamente, debido a las oportunidades para activar espacios de encuentro, diálogo e interacción en su entorno próximo -partiendo de su reconocimiento como sujetos sociales, en tanto, parte de las problemáticas abordadas- como agentes de información, de propuestas y de incidencia sobre los temas abordados.

Finalmente, el tercer aspecto a destacar es la valoración de las y los estudiantes sobre cómo la experiencia en el curso habría incidido en aprender a interactuar positivamente con la diversidad subjetiva.

"El curso trabaja tres [enfoques]...la responsabilidad social universitaria es entablar diálogo y eso implica tolerancia...aquí hemos aprendido a dialogar... buscar rescatar lo que [las personas] han dicho, para empezar a hablar..." (Entrevistada 1).

“...es importante porque en la Feria ya no es que tú solo das información, sino que también la recibes... es algo recíproco...recibes sus puntos de vista $\mathrm{y}$ tratamos de cambiarlos, en el buen sentido para que estén más informados, pero también recibimos sus aportes..." (Entrevistada 7).

Los testimonios realzan cómo la experiencia del curso 
-tanto en los espacios de clase y en la experiencia de trabajos grupales sobre los temas abordados como durante la experiencia de la Feria al presentar sus elaboraciones y dialogar sobre ellas con las y los asistentes- habría incidido positivamente en que reconozcan la importancia de visibilizar y valorar diversas particularidades y subjetividades. De igual manera, en reconocer la existencia de diversas formas de abordar las problemáticas y saberes al respecto -a partir de darse la posibilidad de interpelar sus propios conocimientos-, como abriéndose a generar nuevos aprendizajes considerando el intercambio con diversas aproximaciones, saberes, posiciones y sentires. En tal sentido, los testimonios recogidos destacan la relevancia desde la experiencia del curso con respecto al reconocimiento de las subjetividades de las personas con las que se interactúa en la convivencia, como componente clave para el desarrollo de la empatía como elemento indispensable de la experiencia ciudadana.

\section{| Conclusiones}

El presente trabajo aporta al debate sobre la importancia de fortalecer la RSU como un enfoque ético-político, debido a su potencial para fortalecer el rol de las universidades para asegurar la expansión de una ciudadanía democrática. Entendida esta, más que como un status jurídico, como una identidad política que realza la agencia del sujeto y su capacidad de incidencia en la vida pública; y cuya expansión se asociaría indispensablemente con el fomento de condiciones estructurales e intersubjetivas que garantice una convivencia pública libre y digna de las diversas subjetividades en sociedad.

En tal sentido, y aunque no es posible generalizar las conclusiones del estudio -por ser producto de un estudio de caso y porque la muestra del recojo cuantitativo no fue tomada de manera aleatoria y representativa-, los resultados aportan a desafiar la idea de que incrementar el nivel educativo de las personas o aumentar sus años en el sistema educativo sería suficiente para aportar al desarrollo de disposiciones ciudadanas consideradas importantes para asegurar la legitimidad de la democracia. Particularmente en contextos como el peruano en que dicha relación no se confirma necesariamente (Carrión et al, 2012; 2020). En esa línea, tanto los resultados cuantitativos -que confirman las dos hipótesis abordadas-, como el análisis cualitativo refuerzan la importancia desde la perspectiva de la RSU de continuar indagando sobre los procesos curriculares que explicarían y/o mediarían la relación entre experiencia educativa y la forma en que las y los estudiantes se apropian y ejercen su ciudadanía, como identidad sustantiva de la democracia ${ }^{\text {vi }}$.

Así, aunque es importante, como plantea Vallaeys (2018, p.50), no reducir la apuesta institucional en favor de la RSU a la mera incorporación de contenidos en los programas de formación, consideramos lo desarrollado como aportes para el debate, tanto conceptual como práctico, sobre el carácter particular de la responsabilidad social en los ámbitos universitarios, concretamente con respecto al quehacer de formación.

Los hallazgos reforzarían la importancia de ahondar en la interpelación del rol de la institucionalidad universitaria como agente de socialización política, con un enorme potencial para fomentar disposiciones favorables hacia principios y normas consideradas indispensables para garantizar la legitimidad democrática (Golebiowska, 1995; Hillygus, 2005; Holt \& Tygart, 1969; Van Doorn, 2014). Y en tanto, aspecto indispensable para la transversalización del enfoque de RSU en el quehacer de formación universitaria, aportan en realzar la importancia de analizar aquellos procesos que -más allá de incidir en el desarrollo de capacidades instrumentales- aportarían a que las y los estudiantes desarrollen competencias y compromisos para participar en la vida pública y en favor de un desarrollo sostenible (Juliá, 2015; Pérez, 2009) y 
asociado al bienestar de la ciudadanía en general.

Particularmente, lo presentado refuerza lo destacado por la literatura sobre el potencial de la educación universitaria para fomentar entre sus estudiantes: i) capacidades reflexivas, sentido crítico y de responsabilidad sobre su entorno social y de los problemas estructurales que lo aquejan; ii) mayor apertura hacia la diversidad y cuestionamientos en sus sistemas de valores sobre la restricción de libertades de grupos no hegemónicos; iii) conciencia de su interdependencia como sujetos sociales, y por tanto, preocupados no solo con su bienestar, sino con el sostenimiento de una convivencia que facilite el bienestar de todas las personas; y, iv) que se reconozcan como ciudadanas y ciudadanos con poder público, y por tanto, con capacidad de generar cambios y transformación en favor del bienestar colectivo (Berman, 1990; Cevallos, 2012; Evia, et al, 2017; Gasca-Pliego \& Olvera-García, 2011; Martí, et al. 2014; Stojnic, 2016; Stojnic \& Román, 2016; Wald \& Feinstein, 2011; White, 2013).

Así, el presente trabajo aporta a la discusión sobre el rol de la RSU en la interpelación de la institucionalidad universitaria con respecto a su responsabilidad -más allá de promover el desarrollo de habilidades intelectuales o laborales- en la promoción de un ethos de responsabilidad pública (White, 2013); de una convivencia democrática que fomente la expansión de competencias para una ciudadanía activa y que garantice la expansión del ejercicio de derechos, libertades, dignidad y poder público de todas y todos, desde sus subjetividades. Y así, fortalecer la apuesta por la institucionalización de una responsabilidad social que, en palabras de Pérez (2009, p.49), aporte a que la universidad, como institución social fundamental, se comprometa sostenidamente con incrementar "la ciudadanización democrática de la sociedad".

\section{| Notas}

i A la cual agradezco por el apoyo brindado desde sus direcciones tanto para el trabajo con el profesorado como de responsabilidad social.

ii El instrumento utilizado para el estudio fue adaptado de una encuesta aprovechada por Stojnic y Román (2016).

Así, por ejemplo, en la variable 'Ciudadanía' el valor 0 da cuenta de los resultados de los encuestados antes de empezar el curso y el valor 1 de los resultados luego de culminarlo; y para 'valoración de la participación ciudadana activa', que es una variable de tipo continua, 0 da cuenta del valor mínimo con respecto a dicha actitud y 1 sería el máximo valor posible

iv Por ejemplo: i) tema-participación política; casoestigmatización de protestas y ii) tema-violencia de género; caso-discriminación hacia población trans.

- Es importante destacar la relación de la variable 'interés en la política' con las tres disposiciones analizadas en esta sección, lo cual refuerza lo que la literatura destaca al respecto de dicha disposición política.

vi Aprovecho para reconocer a mi colega y amiga Natalia Consiglieri con quien compartimos tantas jornadas de reflexión $y$ tanto tiempo de compromiso en favor de esta tan importante apuesta.

\section{| Referencias}

Arango, O., Clavijo, S., Puerta, I. \& Sánchez, J. (2013) Formación académica, valores, empatía y comportamientos socialmente responsables en estudiantes universitarios. Revista de la Educación Superior XLIII (1), 169, 89-105.

Berman, S. (1990). Educating for Social Responsibility. Edu- 
cational Leadership, 48 (3), 75-80.

Booth, J. \& Seligson, M. (2009). The legitimacy puzzle in Latin America: Democracy and political support in eight nations. Cambridge University Press.

Carrión, J., Zárate, P. \& Seligson, M. (2012). Cultura política de la democracia en Perú, 2012. Hacia la igualdad de oportunidades. Instituto de Estudios Peruanos.

Carrión, J., Zárate, P., Boidi, F. \& Zechmeister, E. (2020). Cultura política de la democracia en Perú y en las Américas, 2018/19: tomándole el pulso a la democracia. Instituto de Estudios Peruanos.

Cevallos, F. (2012). La responsabilidad social de las instituciones de Educación Superior. Hacia una praxis de ciudadanía responsable. Revista Inclusión Social y Equidad en la Educación Superior, 11 (pp. 105-117).

Chiroleu, A. (2011). La educación superior en América Latina: ¿Problemas insolubles o recetas inadecuadas? Avaliação, Campinas, 16 (3), 631-653.

Cuenca, R. (2018). Las promesas democráticas de la universidad latinoamericana: balance de un centenario. En P., Henríquez (Ed.). El papel estratégico de la educación superior en el desarrollo sostenible de América Latina y el Caribe. (pp. 31-52). UNESCO - ESALC y Universidad Nacional de Córdoba.

De la Cruz, C. \& Sasia, P. (2008). La responsabilidad de la universidad en el proyecto de construcción de una sociedad. Revista Educación y Sociedad, nueva época, 13 (2), 21-51.

Evia, N.; Echevarría, R; Carrillo, C; \& Qunital, R. (2017). Ciudadanía: Análisis de algunos elementos del Modelo de Responsabilidad Social Universitaria en una universidad pública. Revista CS, 23, 77-104.

Gasca-Pliego, E. \& Olvera-García, J. (2011). Construir ciudadanía desde las universidades, responsabilidad social universitaria y desafíos antes el siglo XXI. Convergencia, Revista de Ciencias Sociales, 56, 37-58.

Golebiowska, E. (1995). Individual, value priorities, education, and political tolerance. Political Behavior, 17(1), 23-48. https://doi.org/d5v3ms
Hillygus, S. (2005). The missing link: exploring the relationship between higher education and political engagement. Political Behavior, 27(1), 25-47. https://doi. org/c8q2x9

Holt, N. \& Tygart, C. E. (1969). Political tolerance and higher education. The Pacific Sociological Review, 12(1), 27-33. https://doi.org/h8qg

Juliá, M. (2015). Competencias generales de la formación universitaria: aportes a la calidad con equidad. En G. Carrillo Mendoza (Ed.) El currículo por competencias en la educación superior (pp. 39-62). Pontificia Universidad Católica del Perú.

Ley No. 30220 [Ley Universitaria]. (9 de julio de 2014). Dia-

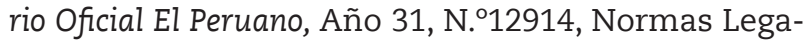
les de Lima, Perú. Ministerio de Educación.

Martí, J., Martí-Vilarb, M. \& Almerich, G. (2014) Responsabilidad Social Universitaria: influencia de valores y empatía en la auto atribución de comportamientos socialmente responsables. Revista Latinoamericana de Psicología, 46(3), 160-168.

Martínez, M. (2006). Formación para la ciudadanía y educación superior. Revista Iberoamericana de Educación, 42, 85-102.

Morrell, M. (2005). Deliberation, Democratic decision-making and Internal Political Efficacy. Political Behavior, 27(1), 49-69.

Mouffe, C. (1999). El retorno de lo político. Comunidad, ciudadanía, pluralismo, democracia radical. Paidós.

Pérez, L. (2009). Formación ciudadana en la educación superior desde la autonomía como competencia comunicativa. La formación profesional en Trabajo Social. Revista Educación y Pedagogía, 21(53), 49-74.

Salazar, L. (2003). La construcción de ciudadanía en educación superior. Revista de Docencia Universitaria, .IV (2), 31-45.

Stojnic, L. (2016). Estudiantes peruanos y el autorreconocimiento de su poder público: ¿cuánto influye la educación universitaria? Debates en Sociología, (43), 5-30.

Stojnic, L. \& Román, A. (2016). Experiencia educativa uni- 
versitaria y tolerancia política: entendiendo la rela-

ción desde el análisis de una muestra de estudiantes peruanos. Revista Internacional de Educación para la Justicia Social, 5(1), 139-160.

Stojnic, L. \& Jungbluth, W. (2018). La Responsabilidad Social Universitaria (RSU) en el Perú: Aproximación Inicial 2016-2017. Red ENARSU.

Unión de Responsabilidad Social Universitaria Latinoamericana [URSULA]. (2019). Investigación Continental Úrsula: Estado del arte de la RSU en América Latina 2019. Red Úrsula. https://cutt.ly/4XQS1Qv

Vallaeys, F. (2008). Responsabilidad Social Universitaria: una nueva filosofía de gestión ética e inteligente para las universidades. Revista Educación y Sociedad, 13 (2), 195-219.

Vallaeys, F. (2014). La responsabilidad social universitaria: un nuevo modelo universitario contra la mercantilización. Revista Iberoamericana de Educación Superior (RIES), V (12), 105-117.

Vallaeys, F. (2018). Las diez falacias de la Responsabilidad Social Universitaria. Revista Digital de Investigación en Docencia Universitaria, 12(1), 34-58. https://doi.org/ $\underline{\mathrm{h} 8 \mathrm{qk}}$

Van Doorn, M. (2014). The nature of tolerance and the social circumstances in which it emerges. Current Sociology Review, 62(6), 905-927. https://doi.org/f6kpt2

Wald, K. \& Feinstein, D. (2011). Higher Education and Political Attitude Change: Is Israel the Exception that Proves the Rule?. American Political Science Association https://doi.org/fxm8sb

White, M. (2013). Higher Education and Problems of Citizenship Formation. Journal of Philosophy of Education, 47(1), 112-127. 